山्山FANÇAISE

$>\mathrm{DE}$

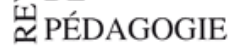

Revue française de pédagogie

Recherches en éducation

$197 \mid 2016$

Les multiples facettes de la créativité dans

l'apprentissage

\title{
Penser l'avenir de manière créative : un enjeu central de l'éducation en vue du développement durable
}

Thinking creatively about the future, a key issue in education for sustainable

development

Alain Pache, Daniel Curnier, Étienne Honoré et Philippe Hertig

\section{(2) OpenEdition}

Journals

Édition électronique

URL : https://journals.openedition.org/rfp/5152

DOI : 10.4000/rfp.5152

ISSN : 2105-2913

Éditeur

ENS Éditions

Édition imprimée

Date de publication : 31 décembre 2016

Pagination : 51-62

ISSN : 0556-7807

\section{Référence électronique}

Alain Pache, Daniel Curnier, Étienne Honoré et Philippe Hertig, «Penser l'avenir de manière créative : un enjeu central de l'éducation en vue du développement durable », Revue française de pédagogie [En ligne], 197 | 2016, mis en ligne le 31 décembre 2019, consulté le 08 janvier 2022. URL : http:// journals.openedition.org/rfp/5152 ; DOI : https://doi.org/10.4000/rfp.5152 


\title{
Penser l'avenir de manière créative : un enjeu central de l'éducation en vue du développement durable
}

\author{
Alain Pache \\ Daniel Curnier \\ Étienne Honoré \\ Philippe Hertig
}

\begin{abstract}
Les auteurs mènent une recherche collaborative sur l'éducation en vue du développement durable (EDD) et la pensée complexe dans six classes vaudoises du degré secondaire 1 (élèves âgés de 12 à 15 ans). Dans un premier temps, ils développent en particulier les liens entre l'EDD, la créativité et la pensée prospective. Dans un second temps, ils montrent comment les élèves pensent l'avenir, sur la base d'une situation fictive portant sur l'aménagement d'un nouveau quartier. Ils constatent que, sans enseignement explicite d'une pensée créative, les solutions d'avenir proposées renvoient généralement à la pensée dominante. Ils terminent en suggérant de favoriser la créativité au niveau de la discipline scolaire, de l'EDD, du modèle d'enseignement-apprentissage et de la matrice historico-spatiale privilégiée.
\end{abstract}

Mots-clés (TESE) : créativité, éducation, développement durable, prévision

\section{Introduction}

L'éducation en vue du développement durable (EDD) correspond à l'insertion contemporaine, dans l'école, d'un projet politique porté par le développement durable (Pache, Bugnard \& Haeberli, 2011). Cependant, le concept de développement durable est controversé sur les plans scientifique et politique (Hertig, 2011), et I'EDD ne fait pas consensus sur de nombreux points, notamment parce qu'elle est issue à la fois de l'éducation à l'environnement et de l'éducation au développement (Varcher, 2011). Les chercheurs ${ }^{1}$ semblent néanmoins unanimes à s'accorder sur les finalités d'une telle éducation, à savoir assurer un avenir convenable aux générations d'ici et d'ailleurs, et, simultanément, engager chaque élève à développer des compétences citoyennes

1 Le masculin est utilisé à titre générique dans ce texte et désigne indifféremment des femmes et des hommes ou des fonctions occupées par des femmes et des hommes. 
lui permettant de trouver sa place dans le monde d'aujourd'hui et d'y agir de manière responsable (Lange, 2013).

Si la prise en charge scolaire, par les sciences humaines et sociales, du passé et du futur n'est pas nouvelle, I'horizon d'attente, lui, semble avoir changé de figure. L'indispensable sauvegarde de la biosphère à laquelle appartient l'espèce humaine et la volonté de répartition équitable des ressources (Audigier, Fink, Freudiger et al., 2011) nous poussent à abandonner l'idéal, rassurant, du futur pour l'injonction plus incertaine de l'avenir. Ces deux termes ne renvoient effectivement pas à la même temporalité : le futur s'inscrirait dans la continuité du passé et du présent, nous engageant dans la direction d'un progrès qui nous attendrait alors que l'avenir serait plutôt ce qui nous fait face et nous appelle, autrement dit une série d'événements qui font rupture avec la pensée dominante et nous obligent à modifier nos perspectives et nos ambitions (Citton \& Latour, 2013).

Ne pouvant nous appuyer sur un passé connu dont le futur serait l'extension, nous sommes contraints à sonder ce qui n'est pas visible dans les conditions du présent, mais qui n'en détermine pas moins ce que nous pourrions devenir (Elias, 1993). Dès lors, il s'agira plus certainement de faire face à la multiplicité des possibles plutôt que de creuser le sillon que le passé nous engage à suivre. La tâche est bien plus ardue.

Dans cette perspective, le rapport au savoir s'en trouve modifié. Ainsi, Citton propose de considérer nos sociétés actuelles comme autre chose que des «économies de la connaissance», mais davantage comme des «cultures de l'interprétation ». Pour lui, cela signifie en effet qu'il faudrait apprendre à interpréter l'information plutôt qu'à l'emmagasiner, mais également dynamiser les disciplines grâce à la «créativité des sous-cultures minoritaires» (2010, p. 123). Rapportées au champ de l'éducation, ces considérations renvoient à la formation du futur citoyen, lequel devra faire preuve de créativité pour résoudre les problèmes du monde de demain. L'école prépare-t-elle aujourd'hui les élèves à ce changement de paradigme? Les dispositifs d'enseignement et d'apprentissage qu'elle propose favorisent-ils suffisamment la créativité des élèves pour qu'ils pensent demain sous des angles différents? Nos élèves sont-ils préparés pour l'avenir?

Après avoir mis en évidence d'un point de vue théorique les enjeux de l'éducation en vue du déve- créativité, les résultats d'une recherche collaborative menée par le Laboratoire international de recherche sur l'éducation en vue du développement durable (LirEDD) de la HEP Vaud seront présentés ${ }^{2}$. Cette recherche vise l'exploration de processus d'enseignement-apprentissage susceptibles de favoriser le développement de la pensée de la complexité et de la créativité chez des élèves des derniers degrés de la scolarité obligatoire (13 à 15 ans).

\section{L'éducation en vue du développement durable : un projet éducatif ambitieux}

Dans la plupart des pays européens, l'éducation en vue du développement durable (EDD) prend depuis quelques années une place importante dans les curricula. En effet, dès leur plus jeune âge et tout au long de leur scolarité, les élèves sont appelés à appréhender des questions environnementales, économiques et sociales et à être éduqués au choix et à la prise de décisions (Orange-Ravachol \& Doussot, 2013). Ces injonctions institutionnelles affirment que l'EDD n'est pas une nouvelle discipline, mais plutôt un objectif transdisciplinaire qui rend nécessaire une recomposition des disciplines (Audigier, 2001; Musset, 2010; Vergnolle Mainar, 2011 ; Pache, 2014). Celle-ci renvoie au moins à cinq enjeux de I'EDD, que nous présentons ci-dessous.

Un premier enjeu de l'EDD consiste à examiner les liens entre les connaissances et l'action. Pour Audigier (2011), il y aurait deux manières de procéder pour initier un travail sur le développement durable : une entrée par des projets d'action (par exemple, les élèves établissent un Agenda 21 pour leur établissement scolaire) et une entrée par l'étude d'une situation ou d'un événement. Dans les deux cas, ce sont les disciplines les mieux à même de répondre aux buts fixés qui seront retenues. Plusieurs recherches ont montré que, dans le premier cas, il existe un risque de rester dans une logique de sens commun, notamment en se limitant au «faire» (Lebatteux \& Legardez, 2011 ; Orange-Ravachol \& Doussot, 2013).

Un deuxième enjeu de l'EDD consiste à identifier les valeurs à la base d'un développement durable : il s'agit de la solidarité, de l'ouverture à l'autre, de la

2 Le présent texte est le résultat d'un travail collectif qui, au-delà des quatre auteurs nommés ici, implique aussi les autres membres de l'équipe en charge de cette recherche au sein du LirEDD. 
justice, de l'égalité ou encore de la responsabilité (Awais, 2016). Lors de la prise de décision et dans l'action, ces valeurs sont souvent en conflit avec d'autres valeurs comme le profit, le plaisir ou encore la préservation des acquis. Plus fondamentalement, il apparaît nécessaire de discuter de manière explicite le modèle de durabilité qui sous-tend I'EDD : est-on dans une durabilité faible (qui favorise la croissance) ou vise-t-on vraiment un changement en profondeur par une durabilité forte?

Un troisième enjeu consiste à développer la pensée complexe. Les recherches en didactique consacrées à cet enjeu sont encore relativement rares. Relevons cependant une série de travaux portant sur le développement d'une compétence systémique (Ossimitz, 2000; Assaraf \& Orion, 2005; Frischknecht-Tobler, Nagel \& Seybold, 2008; Rempfler, 2009, 2010; Rempfler \& Uphues, 2012). Celle-ci consiste notamment à décrire un système sous la forme d'un schéma fléché et à utiliser ce système en vue de formuler des pistes d'action. Dans le contexte francophone, d'autres chercheurs s'appuient sur les travaux de Morin $(1999,2000)$ pour construire un modèle basé sur cinq dimensions : des relations de causalité, des boucles de rétroaction (ou de récursivité), des tensions dialogiques, des relations fondées sur le principe hologrammique $^{3}$ et des relations fondées sur des modes de pensée disciplinaires (Jenni, Varcher \& Hertig, 2013). Ces chercheurs montrent que cette pensée complexe s'élabore, dans le cadre d'un débat, le plus souvent dans les interactions entre les élèves ou entre l'enseignant et les élèves. Ils constatent en outre que de tels outils permettant de penser la complexité n'ont dans la plupart des cas pas fait l'objet d'un travail explicite avec les élèves.

Un quatrième enjeu consiste à développer la capacité à construire le problème sur lequel travailler. En effet, le monde d'aujourd'hui étant problématique (Fabre, 2011), il s'agit de proposer aux élèves des outils leur permettant d'analyser les situations qu'ils rencontrent et d'apprendre à poser des questions critiques et réflexives. Dès lors, comme le rappellent Janzi et Sgard (2013), la réflexion porte avant tout sur le choix du problème, sur l'insertion dans un paradigme, sur le choix des situations-problèmes, des dispositifs de controverses, de débat ou des médias. Certaines études empiriques montrent l'importance de l'élément déclencheur et de la démarche de déconstruction d'un

3 Le principe hologrammique consiste à affirmer que le tout est déjà inscrit dans la partie, de la même façon que la partie est inscrite dans le tout. objet de savoir (Humbel, Jolliet \& Varcher, 2013). D'autres insistent sur les différents «versants» de l'activité de problématisation. Laplace (2006, p. 163) en identifie trois : le versant «flottant», qui consiste à ouvrir le questionnement; le versant "structurant", qui consiste au contraire à trier les propositions et à ne retenir que celles qui permettent d'identifier un réel problème; et le versant "créatif», qui, en appui du versant structurant, consiste à favoriser la relance du questionnement.

Le cinquième enjeu est celui que nous allons développer plus largement dans cet article. II consiste à développer la pensée prospective, autrement dit la capacité à imaginer non seulement un avenir, mais des avenirs possibles. Comme le rappelle Audigier (2011), il s'agit dès lors de prendre en compte la société du risque (Beck, [1986] 2001), I'incertitude (Callon, Lascoumes \& Barthe, 2001) ou encore la prospective (Gaudin, 1990). Cette prise en compte de la liberté humaine doit être introduite dans l'enseignement comme une dimension nécessaire pour raisonner le développement durable et agir.

Développer la pensée prospective signifie aussi faire preuve d'invention. Pour Citton, qui reprend les cours de Deleuze interprétant Bergson, la capacité $d$ 'invention repose «sur une dilatation du circuit qui va de la perception à la réaction motrice » et «émerge de l'intervalle qui s'est inscrit entre les deux». Dès lors, la réaction est «nouvelle et imprévisible» (2010, p. 54-55). L'esprit opère donc par «des sauts entre différents niveaux de réalité, il s'y oriente par des pressentiments tâtonnants, il se trouve surpris par des coïncidences et des superpositions de lignes qui s'imposent soudain avec une évidence anticipant et excédant tout savoir » (2010, p.56).

Dans le cadre de l'enseignement de I'EDD, il est demandé aux élèves de penser l'avenir, d'inventer un monde qui est différent de celui dans lequel ils vivent. Pour ce faire, ils doivent s'appuyer sur ce qu'ils connaissent du présent et du passé. Les savoirs construits dans le cadre scolaire sont donc des ressources que les élèves vont mobiliser au même titre que les savoirs provenant de la famille, des médias ou du monde associatif (Audigier, Fink, Freudiger et al., 2011). L'élève ne devrait ainsi pas reproduire à l'identique ce qu'il connaît, de sorte que l'on pourrait parler d'«invention interprétative» (Citton, 2010, p.58).

De ce point de vue, le concept d'invention semble relativement proche du concept de créativité. C'est ce que nous allons explorer dans la section suivante. 


\section{Créativité et éducation en vue du développement durable}

Le concept de créativité apparaît sur un plan politique dans la Déclaration de Rio de 1992 sur l'environnement et le développement :

Il faut mobiliser la créativité, les idéaux et le courage des jeunes du monde entier afin de forger un partenariat mondial, de manière à assurer un développement durable et à garantir à chacun un avenir meilleur (Principe 21 de la Déclaration de Rio sur l'environnement et le développement).

Dans ce texte, le terme de "créativité » est envisagé avec un sens proche du sens commun - le pouvoir de création, $d^{\prime}$ invention ${ }^{4}$. Pour notre part, nous nous référons aux travaux menés en psychologie, et notamment à la définition de Lubart, Mouchiroud, Tordjam et alii, pour qui la créativité est «la capacité à réaliser une production qui soit à la fois nouvelle et adaptée au contexte dans lequel elle se manifeste» $(2003$, p. 10).

Ces deux idées de nouveauté et d'adaptation au contexte peuvent apparaître contradictoires. En effet, elles correspondent à deux temporalités différentes: celle de l'événement (ou de l'action), et celle qui juge de l'adéquation au contexte. Autrement dit, une production peut, sur le moment, sembler hors contexte, mais peut s'avérer pertinente quelques années après. Mais, plus généralement, la créativité, en tant que telle, "crée » de la temporalité : la nouveauté détermine un passé (à dépasser) et des conséquences qui obligent un futur. Dans une perspective didactique, de tels enjeux ne sauraient être éludés et nécessitent un travail progressif. II s'agit, notamment, de permettre aux élèves d'identifier, dans un champ disciplinaire ou dans un problème qui dépasse le cadre disciplinaire, ce qui relève de la nouveauté.

Une deuxième difficulté provient du fait qu'il n'existe pas, ni dans la société, ni à l'école, de norme permettant de juger de la créativité d'une production. Dès lors, un consensus social doit être établi (Lubart, Mouchiroud, Tordjam et al., 2003), notamment afin de clarifier les dynamiques temporelles mentionnées plus haut. Enfin, il convient de distinguer une production ou une conduite créative au regard de l'histoire de l'humanité - la créativité historique -, et une production qui peut être considérée comme novatrice par rapport aux précédentes expériences de l'individu - la créativité psychologique (Boden, 2005). Dans le but de favoriser le développement d'une «pensée de la créativité», il importe de baliser suffisamment les démarches, de manière à ce que l'élève identifie ce qui est nouveau dans les démarches et modes de pensée mis en œuvre.

Depuis plusieurs années, les chercheurs en psychologie définissent la créativité selon une « approche multivariée» (Lubart, 2012, p. 15). En effet, cette capacité dépendrait de facteurs cognitifs, de facteurs conatifs et de facteurs environnementaux. Dans le domaine de la cognition, le chercheur parle d'«encodage sélectif» lorsque l'individu relève dans son environnement une information en lien avec le problème qu'il doit résoudre. Par exemple, un chercheur a constaté un jour que l'énergie du vent (produite par la respiration) pouvait être convertie en électricité. II a ainsi inventé un masque permettant de recharger de petits appareils électroniques comme les lecteurs MP3. Si I'on transfère un tel exemple dans le contexte de l'enseignement, il faut donc reconnaître, avec Perrin, qu'il est nécessaire de laisser une certaine liberté à l'élève sans toutefois le laisser faire n'importe quoi : il s'agit en effet "de délimiter des espaces de possibles et d'en interdire d'autres» (2014, p. 10). Dit autrement, cela signifie que les tâches proposées aux élèves doivent à la fois présenter des contraintes et favoriser les solutions multiples (notamment parce qu'elles s'appuient sur plusieurs ressources).

Dans le Plan d'études romand (PER), en Suisse, c'est le terme de «pensée créatrice » qui est utilisé, dans l'idée de développer la "pensée divergente», de reconnaître "sa part sensible» et de concrétiser «l'inventivité» ${ }^{5}$. D'une certaine manière, une telle approche transversale vise à critiquer une école centrée sur le conformisme et la pensée convergente (Lubart, Mouchiroud, Tordjam et al., 2003). Mais elle vise aussi à combattre certaines représentations qui consistent à dire que la créativité est étroitement liée à une dimension artistique, éludant du même coup sa présence dans d'autres disciplines (Schumacher, Coen \& Steiner, 2010).

Dans une perspective de didactique des sciences humaines et sociales, nous pensons pouvoir mobiliser de la créativité à la fois dans la mise en œuvre d'un point de vue disciplinaire et en faisant dialoguer les disciplines entre elles afin de créer de nouveaux points de vue. À l'école, nous retrouvons donc cette

5 Comme l'ont montré Puozzo Capron et Martin (2014), il faudrait plutôt parler de "pensée créative», car l'élève peut créer un produit sans avoir à être créatif. 
distinction entre créativité historique et créativité psychologique, dès lors que les points de vue disciplinaires sont en cours d'élaboration chez les élèves. Par ailleurs, les disciplines peuvent être vues comme des points d'appui pour entrer dans des logiques qui les transcendent (Vergnolle Mainar, 2011).

Afin de prolonger la réflexion, nous pouvons mentionner les dispositifs qui favorisent la pensée divergente, autrement dit qui mettent en avant la multiplicité des possibles et l'idée selon laquelle le présent lui-même n'est plus qu'un possible. Il s'agit des débats (Audigier, Fink, Freudiger et al., 2011), des situationsproblèmes (Fabre, 2009), des jeux de rôles et de simulation ou encore des projets. Dans de tels dispositifs, les élèves sont mis dans des situations proches de situations sociales et l'enseignant joue un rôle central par ses relances et ses diverses incitations à la réflexion.

Enfin, au carrefour entre la créativité et le développement durable, il nous paraît intéressant de mentionner les trois niveaux de changement qui pourraient baliser les apprentissages selon Sterling (2011, p.25). Ces derniers seront par ailleurs utiles pour nos analyses : le changement "conformatif» (doing things better), « réformatif» (doing better things) et "transformatif» (seeing things differently). Le changement «conformatif» consisterait par exemple à développer un moteur de voiture consommant moins d'essence. Le changement "réformatif» consisterait à créer un moteur hybride, fonctionnant à l'essence et à l'électricité. Enfin, le changement «transformatif » consisterait à changer de paradigme, ce qui reviendrait, dans notre exemple, à donner la priorité à des manières de se déplacer autres que le véhicule individuel (par exemple en transports publics), et à repenser la mobilité.

En nous référant à la distinction préalablement posée entre futur et avenir (Citton \& Latour, 2013), nous pourrions dire que le changement "transformatif» implique de s'appuyer sur une logique différente de celle du présent.

En l'état actuel de nos réflexions, la créativité apparaît comme une première étape pour penser l'avenir. L'invention de l'avenir interviendrait dans un second temps, avec l'idée de scénarisation. En effet, si l'on reprend l'exemple précédent, on peut être créatif en proposant le covoiturage. En revanche, trouver des moyens permettant d'implanter véritablement le covoiturage dans la société relèverait plus de l'invention de l'avenir ou de la scénarisation. Le passage par l'écrit, ou la mise en récit, s'avère donc être l'étape ultime pour décrire un monde nouveau.

\section{Méthodologie de la recherche}

Les données analysées ont pour l'essentiel été recueillies lors d'entretiens semi-dirigés avec des groupes d'élèves (focus groups) issus de six classes vaudoises du degré secondaire 1 (élèves âgés de 12 à 15 ans) et menés après une séquence d'enseignement-apprentissage portant sur une thématique étudiée dans une perspective d'EDD. Il avait notamment été demandé aux enseignants partenaires de mettre l'accent sur les outils de pensée permettant l'apprentissage de la complexité. D'autres données consistent en des enregistrements vidéo de moments de ces séquences et des productions d'élèves ${ }^{6}$; nous avons également conduit des entretiens ante et post avec les enseignants. Une partie seulement de ces données a été exploitée en vue de la présente contribution (entretiens en focus groups, entretiens ante et post). On notera encore que les enseignants partenaires de cette recherche collaborative ont tous plusieurs années d'expérience; ils enseignent les sciences naturelles, l'économie, l'histoire, la géographie et l'éducation à la citoyenneté.

Nous avons élaboré une situation fictive (qui pourrait cependant être utilisée comme situation d'enseignement-apprentissage en classe) et l'avons proposée aux élèves au début de l'entretien semi-dirigé en focus group. Nous attendons de ce dispositif qu'il nous permette d'analyser les outils de pensée complexe mobilisés par les élèves ainsi que la pensée créative. Voici une brève description de cette situation.

Il y a actuellement un sérieux manque de logements à loyers modérés dans la région lémanique. II se trouve qu'un important projet de construction d'un quartier d'habitation à loyers modérés dans la commune de $\mathrm{NNN}^{7}$ va être examiné prochainement :

- ce quartier compterait environ 250 logements de 3,4 ou 5 pièces, donc destinés surtout à des familles; - vu qu'il s'agirait d'un quartier d'habitation à loyers modérés, l'idée est qu'il permette d'instaurer une certaine "mixité sociale» dans la partie de la commune où il serait construit; il serait en effet proche d'un quartier de villas;

- la zone où il pourrait être construit est une zone où la construction est autorisée;

6 Les données ont été récoltées pendant l'année scolaire 20122013 , dans les classes d'un premier groupe de 7 enseignants partenaires. Le recueil de données s'est poursuivi en 2013-2014 dans les classes d'un deuxième groupe de 8 partenaires, puis 2014-2015 dans les classes d'un dernier groupe de partenaires. Certaines des données recueillies sont encore en cours d'analyse au moment où la version définitive de ce texte est soumise (septembre 2016).

7 C'est une commune connue des élèves qui a été mentionnée. 
- le projet soulève toutefois quelques problèmes :

- construire les bâtiments du quartier et les accès (routes, chemins, parkings) suppose de faire disparaître un secteur dont le couvert végétal (la végétation «naturelle») est riche et diversifié et offre des abris pour la faune (mammifères, oiseaux, batraciens, reptiles, insectes). Ce secteur n'est toutefois pas protégé par des dispositions légales;

- dans le secteur concerné se trouvent deux bâtiments assez anciens et inutilisés, mais dont l'architecture est digne d'intérêt; ces bâtiments, qui ne sont pas protégés par la loi, devront être démolis;

- s'il est construit, ce quartier d'habitation va forcément engendrer des déplacements pour ses habitants, lorsqu'ils devront se rendre à leur travail, aller à l'école, faire des courses, etc.;

- il y a certes un terminus d'une ligne de transports publics à quelques minutes à pied du quartier, mais on sait que les gens ont souvent tendance à se déplacer au moyen de véhicules privés plutôt qu'avec les transports publics.

Dans le cadre des entretiens, il a été demandé aux élèves de se positionner par rapport à la construction de ce quartier en argumentant leur point de vue et en proposant, éventuellement, des solutions pour minimiser les problèmes.

Les entretiens se sont déroulés dans des conditions proches de ce qui est pratiqué dans des focus groups (Markova, 2003). Ces derniers ont pour but de traduire le fonctionnement d'une "société pensante en miniature» (Markova, 2003, p. 223). Autrement dit, le dispositif permet d'accéder aux représentations des élèves, mais également aux processus d'élaboration de la pensée sociale. À cette fin, deux groupes de trois élèves (trois garçons et trois filles) ont été constitués dans chaque classe, dans le but de faire émerger des visions d'avenir relatives à la situation proposée. II était prévu que le chercheur n'intervienne que de manière limitée durant l'entretien, favorisant ainsi les échanges, la confrontation d'idées et le développement des arguments. Il a en outre été précisé que le contenu des discussions ne serait pas rapporté aux enseignants et que ces dernières ne feraient pas l'objet d'une évaluation.

Pour analyser ces entretiens, nous avons élaboré les trois ensembles de mots-clés qui apparaissent dans le tableau 1 ci-dessous. Il s'agit de catégories qui ont pour but de préciser les acteurs concernés, les actions qu'ils pourraient mener et, enfin, la temporalité de ces actions. À ce stade, nous nous appuyons sur divers travaux de sciences sociales pour faire l'hypothèse que ces catégories pourraient permettre de faire émerger une pensée de la créativité (Callon, Lascoumes \& Bar-
Le premier ensemble permet de distinguer l'acteur indifférencié (11), I'acteur familial (12), l'acteur individuel (13) et l'acteur collectif (14). Le deuxième ensemble distingue l'action présentée comme une solution, une vérité ou une décision (21), l'argument présenté comme une explication ou une justification (22), la condition (23) et une prise de conscience autour de la difficulté à décider ou à agir (24). Le troisième ensemble, enfin, permet de distinguer des temporalités : le présent (31), le futur (32), I'association présent-futur (33) et le passé (34).

Nous avons réalisé notre analyse à l'aide du logiciel Transana ${ }^{8}$, qui permet de regrouper tous les éléments de notre corpus et de faire des recherches par mots-clés.

Ainsi, dans un premier temps, l'entretien a été découpé en épisodes. Il s'agit d'extraits regroupant au maximum 25 lignes de transcription et présentant une unité de sens. Dans un deuxième temps, un ou plusieurs mots-clés ont été attribués à chaque épisode.

Une fois que tous les épisodes ont été codés, le logiciel permet d'effectuer une recherche par mot(s)clé(s) et fournit des rapports d'analyse.

En ce qui concerne les entretiens enseignants (ante et post), en vue de cet article, nous avons centré notre analyse sur le troisième ensemble de mots-clés.

\section{Présentation des résultats}

\section{Propos d'élèves}

Une analyse systématique portant sur les mots-clés 32 «futur» et 33 «présent-futur» nous permet de dégager un certain nombre d'idées créatives en lien avec la situation proposée. Les élèves sont généralement conscients du manque de logements dans l'agglomération lausannoise et sont, dans l'ensemble, favorables à la construction d'un nouveau quartier. Toutefois, ils relèvent certaines conditions. Environ la moitié des élèves s'opposent à la destruction de bâtiments anciens et proposent soit de les utiliser pour en faire un musée, soit de les déplacer dans un autre lieu. Cette stratégie du «déplacement» est aussi suggérée pour combler la perte d'un écosystème. Autrement dit, les élèves proposent de «replanter» ailleurs. En ce qui concerne les problèmes de transport, un élève propose des subventions communales afin de faire baisser le prix des abonnements, alors que trois autres élèves

8 Ce logiciel a été créé par Chris Fassnacht au Wisconsin Center for Education (WCER), à Madison. Il est disponible en ligne : <http:// www.transana.org/> (consulté le 28 février 2018). 
suggèrent de prolonger certaines lignes de bus, afin de mieux desservir le quartier. Quatre élèves proposent un changement des modes de vie, à savoir favoriser les colocations, le covoiturage, les liens avec d'autres «styles de vie» et s'habituer à occuper des logements plus petits. Deux autres élèves, enfin, proposent de "s'arranger avec les paysans» pour développer des zones constructibles supplémentaires, créer des quartiers sans voitures, utiliser les impôts pour préserver des écosystèmes, subventionner certaines familles, ou encore créer une nouvelle ville «dans des endroits où il n'y a pas une énorme végétation ».

Peu d'élèves proposent une réflexion sur l'importance de distinguer diverses échelles temporelles. Les solutions qu'ils proposent sont donc toutes situées dans un futur proche, qui peut se situer soit dans le prolongement du présent, soit en rupture avec ce même présent. Cette question, d'ailleurs, est souvent peu claire dans les discours analysés. Lorsque ce point est précisé, c'est généralement pour amener l'idée du changement et de la nouveauté, comme l'illustre l'extrait 1 ci-dessous :

Extrait 1 (entretien E1, classe 1):

Chercheur : D'accord. Et puis si vous trouviez en face de vous quelqu'un qui vous disait "oui mais on sait très bien que ça va augmenter les transports individuels, les trajets dans la région de NNN, qui est déjà surchargée au niveau des trajets routiers», qu'est-ce que vous lui diriez?

Élève 1 : Ben dans tout le monde entier c'est pareil, on s'habitue au changement. Il faudra prendre des mesures par rapport à ça, mais ça c'est depuis le début de l'univers. On fait tout le temps des changements. II faut penser un tout petit peu à la nouveauté et si on est obligé de faire ça, parce qu'on est obligé, y a vraiment une très grande crise, ben il faudra peut-être faire des nouvelles infrastructures, que ce soit peut-être routier ou j'en sais rien mais le changement ça fait partie de la vie donc il faudra faire des choses évidemment.

Nous avons toutefois remarqué un élève qui pose le problème à différentes échelles. Selon lui, une réflexion à court terme pencherait en faveur de la construction de nouveaux quartiers, alors qu'une réflexion à long terme devrait plutôt nous inciter à préserver la biodiversité. Le dilemme ainsi posé, il se positionne tout de même en faveur de la biodiversité (extrait 2) :

Extrait 2 (entretien E2, classe 4) :

Élève 1 : Ben moi je dis qu'à court terme, ça va être extrêmement utile pour les, ben les gens qui ont besoin de ces logements-là, de les construire. Mais à long terme, ben le fait de les construire, ça va un petit peu détruire l'écosystème local qui peut détruire d'autres écosystèmes lui-même en se détruisant, donc à long terme, ça va les desservir plutôt qu'autre chose, donc c'est pas une super idée, vaut mieux réfléchir à long terme que à court terme comme on le fait. 
Chercheur: D'accord. Mais si on réfléchit à long terme, quelle décision on prendrait?

Élève 1 : Si on réfléchit à long terme...

Élève 2 : Construire ailleurs.

Chercheur: Et puis les gens vont vivre où?

Élève 1 : Ben ailleurs.

Élève 2 : Si on, si on réfléchit à long terme, on, je sais pas, imaginons, on pourrait, j'ai entendu, enfin il y a des endroits par exemple où il n'y a pas une énorme végétation comme ça, et ces endroits-là, on pourrait créer une nouvelle ville par exemple...

II est rare que les élèves fassent référence de manière explicite à des savoirs construits dans le cadre scolaire. Or, nous savons que ceux-ci jouent un rôle pour penser une nouvelle situation (Audigier, Fink, Freudiger et al., 2011), mais qu'ils sont mêlés à des savoirs sur le monde provenant d'ailleurs (Tutiaux-GuilIon, 2008). Nous pouvons ainsi dire que les propositions formulées ne traduisent qu'un processus d'«invention interprétative» (Citton, 2010) relativement opaque. En effet, les élèves ne parviennent généralement pas à montrer le lien entre ce qui a été appris et ce qui est nouveau. Dans le corpus étudié, seul un élève fait explicitement référence à un travail réalisé en classe (la présentation d'un exposé en l'occurrence). L'extrait 3 rapporte ses propos:

Extrait 3 (entretien E2, classe 4) :

Élève 1 : Et puis après, le fait de construire des bâtiments, moij'avais parlé, dans mon exposé, je parlais de construire une station de ski. Et puis ça engendre plein de pollution, ça engendre de la pollution parce qu'après, les gens ils se déplaceront, même s'ils utilisent des transports en commun, certes ça consomme moins, mais ça consomme quand même, parce que faut genre apporter des, vous savez, les lignes électriques. Et puis voilà, après, après peut-être que, si, si ça devient, si ça marche vraiment, il y aura peut-être une gare, ça encore, ça polluera encore, etc., etc. Et ben après, ce qui concerne les plantes, faudrait, faudrait voir, limite créer, je sais pas moi, genre, garder, préserver au centre, imaginons, je sais pas faire, un, enfin...

Chercheur : une zone.

Élève $1:$... une zone, et dans cette zone, et ben il y aurait une partie réservée à l'écosystème, par exemple, ben un peu comme Central Park à New York, bon en plus petit certes, mais...

Chercheur: D'accord. Vous connaissez bien Central Park?

Élève 1 : Oui.

Chercheur : Ça vous semble un écosystème équilibré, naturel?

Élève 2 : Alors, oui et non, parce qu'il y a des zones qui sont franchement préservées, interdites au public, et
Notons que ces propos illustrent bien le processus d'invention interprétative évoqué plus haut. II n'est pas possible de savoir si la référence à Central Park se fonde sur une expérience vécue, sur des images vues dans les médias ou si elle renvoie à un apprentissage scolaire. En tout état de cause, les propositions de cet élève se caractérisent par un mixte de sens commun et d'éléments dont on peut dire qu'ils commencent à mettre le sens commun à distance. On peut aussi relever, en référence aux catégories définies par Sterling (2011) pour qualifier le changement, que ces propositions semblent appeler des changements de type « réformatif ». Pour reprendre la distinction entre futur et avenir évoquée au début de ce texte, on peut considérer que ces propos sont plutôt ceux d'élèves qui se projettent dans le futur et non dans l'avenir.

En guise de synthèse, nous pouvons donc dire qu'une majorité d'élèves sont capables d'imaginer des solutions futures, ce qui constitue une première étape dans l'élaboration d'une pensée prospective. Ils peinent toutefois à mentionner un éventail d'avenirs possibles. En outre, nous avons constaté que les élèves n'évaluent pas spontanément le caractère créatif de leurs propositions. Selon nous, trois hypothèses pourraient l'expliquer. D'une part, la situation sociale proposée ne leur demande pas nécessairement de formuler des propositions créatives. D'autre part, nous ne sommes pas certains que les élèves parviennent à distinguer ce qui relève d'une pensée sociale dominante ${ }^{9}$, qu'il s'agirait de cartographier, de ce qui constitue une pensée sociale créative. Enfin, nous pensons qu'une pensée créative en tant que telle n'a pas été enseignée de manière explicite. C'est cette troisième hypothèse que nous allons explorer dans la sous-section suivante.

\section{Propos des enseignants partenaires}

Outre les discours oraux des élèves, nous avons analysé les propos des enseignants partenaires lors des entretiens ante et post, afin d'évaluer dans quelle mesure ils font eux-mêmes référence au futur et à l'avenir, d'une part, et à des dispositifs favorisant le processus de créativité, d'autre part. Nous avons procédé de la même manière que pour les élèves, en codant les entretiens à l'aide des mots-clés 32 «futur» et 33 «présent-futur».

9 Par «pensée sociale», nous entendons à la fois la manière donc chacune et chacun construit une pensée de la vie sociale, et ce qu'elle ou il utilise pour penser et communiquer avec les autres, que ce soit par oral ou par écrit (Audigier \& Haeberli, 2004). 
D'une manière générale, lorsque les enseignants partenaires s'expriment sur les enjeux de l'éducation en vue du développement durable, ils mentionnent la nécessité de se projeter dans le futur, à court et à long terme, par exemple en prenant conscience des «conséquences de nos actes » ou en construisant «une vision de là où [les élèves] voudraient aller». Les enseignants partenaires font part d'une préoccupation pour les "générations futures », relayant ainsi un discours social dominant. Ils sont généralement conscients de l'évolution des savoirs, des changements dans les «façons de penser et de concevoir», par exemple en lien avec l'idée de progrès, comme le mentionne l'enseignant de la classe 1 :

\section{Extrait 4 (N., classe 1) :}

Vraiment de voir une fois plus loin, de se dire, pas parler en terme de 10 ans, 20 ans mais dans 50 ans, dans 100 ans, se rendre compte aussi des conséquences de nos actes qu'il peut y avoir sur notre façon de fonctionner qu'on n'avait peut-être pas auparavant où on était plus dans une science de développement, sans se rendre compte peut-être des conséquences. Mais on se disait c'est le progrès alors que maintenant est-ce que le progrès est toujours bénéfique? Et on arrive toujours à ce dilemme de se dire progrès c'est bien mais en même temps, quel impact ça a sur l'ensemble de la planète et puis du fait de vivre ensemble, d'essayer d'être tous des êtres humains en fait.

Nous avons constaté que les sept enseignants partenaires intègrent, dans leur enseignement, des dispositifs favorisant la créativité (jeu de simulation, exposés, débats, par exemple). Une majorité d'enseignants ne mentionnent toutefois pas la créativité comme un enjeu d'apprentissage (cinq sur sept). Dès lors, c'est un peu comme si la créativité - que l'on considère implicitement comme une capacité transversale - ne devait pas faire l'objet d'un enseignement spécifique. Ainsi, ce serait en se confrontant à la situation que I'on deviendrait créatif.

Dans le cas des enseignants partenaires qui mentionnent la créativité (et plutôt la capacité à imaginer et l'invention), il s'agirait, pour l'un, d'avoir "cette liberté d'imaginer tous les développements possibles» [au sujet des tablettes numériques] et d'avoir ainsi des "outils» pour être des citoyens responsables. En revanche, pour le second, il s'agirait de s'appuyer sur un savoir disciplinaire pour l'adapter à une situation tirée de «l'imaginaire» (extrait 5) :

Extrait 5 (S., classe 4) :

Alors, il y a tout d'abord une phase de la séquence dans laquelle il y a du savoir disciplinaire qui va être concep- tualisé, expliqué, explicité. Ensuite, ily a une phase dans laquelle les élèves vont devoir prendre ce savoir et le transformer en inventant quelque chose, donc en, en, en mettant en corrélation quelque chose de l'imaginaire auquel ils doivent adapter le savoir disciplinaire qu'ils ont appris ${ }^{10}$. Et dans une troisième phase, ils vont avoir des documents à disposition dans lesquels ils vont devoir chercher des informations spécifiques à des cas réels, des situations réelles de, de plantes exotiques et envahissantes ou pas, donc c'est à ce moment-là qu'ils vont pouvoir faire la distinction. Et en même temps, compiler les, les, les informations recherchées dans les documents qui seront à leur disposition, les informations utiles à la présentation d'une telle espèce au reste de la classe. Donc, y a, y a vraiment différentes phases dans lesquelles les élèves prennent possession du savoir, le mettent en pratique, vont rechercher, faire des recherches documentaires, et ensuite présentent ce qu'ils ont obtenu au reste de la classe.

Notons toutefois que ni l'un ni l'autre de ces deux enseignants partenaires ne fait de cette capacité à inventer un objet qui va donner lieu à une institutionnalisation.

\section{En guise de propos conclusifs}

Les analyses et les résultats dont il est fait état dans cet article s'inscrivent dans une recherche plus large, dont l'objet porte en premier lieu sur les «outils de pensée» que les élèves devraient s'approprier pour être en mesure d'appréhender des questions sociétales complexes liées aux enjeux du développement durable. Par "outils de pensée», nous entendons en particulier des concepts - disciplinaires ou interdisciplinaires - qui permettent d'organiser les connaissances (Barth, [1987] 2001 ; Bruner, [1996] 2008; Morin, 1999; Hertig, 2009).

Dans ce contexte, des réflexions sur la capacité des élèves (et des enseignants) à penser le futur et, $a$ fortiori, l'avenir constituent selon nous un passage obligé. Mais penser l'avenir consiste aussi à proposer des solutions en rupture avec la pensée dominante. Encore faut-il pouvoir identifier cette pensée dominante. Sur la base de celle-ci, on pourrait imaginer une tâche où les élèves seraient amenés à proposer d'autres solutions, à la fois créatives et réalistes, à un problème complexe.

\footnotetext{
10 C'est nous qui soulignons. Les élèves devaient produire un poster présentant de manière synthétique l'impact écologique de l'implantation d'une espèce végétale invasive imaginaire et les moyens de l'éradiquer. La démarche, située au début du processus d'enseignement-apprentissage, avait pour but de motiver les élèves, mais également de les amener à se questionner, à construire une problématique et les hypothèses qui y sont liées.
} 
Les constats dont nous avons fait état montrent que les élèves n'évaluent pas spontanément le caractère créatif de leurs propositions. Pourtant, les solutions qu'ils proposent sont souvent à la fois nouvelles et adaptées au contexte (Lubart, Mouchiroud, Tordjam et al., 2003), ce qui nous conduit à dire qu'ils sont capables de faire preuve de créativité. Cependant, dans la mesure où ils ne sont pas habitués à ce genre d'exercice, leur capacité à scénariser et à inventer demain semble limitée. En effet, du côté des enseignants, les dispositifs proposés favorisent la pensée divergente, mais un enseignement explicite du processus créatif n'est généralement pas mis en œuvre - mis à part chez deux enseignants partenaires -, comme s'il suffisait d'être placé dans une situation complexe pour développer sa créativité. De tels constats rejoignent des travaux antérieurs qui montrent que la créativité est souvent associée à une dimension artistique, et très peu à des enseignements disciplinaires (Schumacher, Coen \& Steiner, 2010).

Plus généralement, les réflexions dont nous rendons compte dans cet article nous ont fait prendre conscience du fait que la créativité n'est pas un objet d'apprentissage en soi. Les analyses menées sur d'autres pièces du corpus vont dans ce sens et nous conduisent à estimer que la créativité serait plutôt une capacité transversale apparaissant potentiellement et, souvent, "naturellement» au niveau de la discipline scolaire, au niveau de l'éducation en vue du développement durable, au niveau du modèle d'enseignement

\section{Bibliographie}

ASSARAF O. \& ORION N. (2005). «Development of system thinking skills in the context of Earth system education ». Journal of research in science teaching, $n^{\circ} 45(5)$, p. 518-560.

AUDIGIER F. (2001). «Les contenus d'enseignement plus que jamais en question ». In C. Gohier \& S. Laurin (dir.), Entre culture, compétence et contenu. La formation fondamentale, un espace à redéfinir. Québec : Éditions logiques, p. 141-192.

AUDIGIER F. (2011). «Éducation en vue du développement durable et didactiques». In F. Audigier, N. Fink, N. Freudiger \& P. Haeberli (dir.), L'éducation en vue du développement durable: sciences sociales et élèves en débats. Genève : Université de Genève, p. 47-71.

AUDIGIER F., FINK N., FREUDIGER N. \& HAEBERLI P. (dir.) (2011). L'éducation en vue du développement durable : sciences sociales et élèves en débats. Genève : Université de Genève. et d'apprentissage que I'on favorise, ou encore au niveau de la matrice historique et spatiale que l'on privilégie (Di Méo \& Buléon, 2005).

Enfin, ces réflexions nous ont aussi amenés à élargir notre conception des «outils de pensée» qui sont I'un des objets centraux de notre recherche: considérer notre corpus sous l'angle de la créativité nous conduit à ne pas limiter la notion d'«outil de pensée» au registre cognitif, aux concepts centraux des disciplines mobilisées. La créativité apparaît en effet comme un outil de pensée au fort potentiel heuristique.

Alain Pache

Haute École pédagogique du canton de Vaud, UER Didactiques des sciences humaines et sociales, LirEDD alain.pache@hepl.ch

Daniel Curnier

Haute École pédagogique du canton de Vaud, UER Didactiques des sciences humaines et sociales, LirEDD curnier.daniel@gmail.com

Étienne Honoré

Haute École pédagogique du canton de Vaud, UER Didactiques des sciences humaines et sociales, LirEDD etienne.honore@hepl.ch

Philippe Hertig

Haute École pédagogique du canton de Vaud, UER Didactiques des sciences humaines et sociales, LirEDD philippe.hertig@hepl.ch
I'histoire, de la géographie, de la citoyenneté. Actes du Colloque international «Journée d'études des didactiques de l'histoire et de la géographie», IUFM de Caen.

AWAIS N. (2016). Aux valeurs! Peut-on développer la pensée critique et la responsabilité des adolescents dans le cadre scolaire? Réflexions didactiques. Saint-Denis : Edilivre.

BARTH B.-M. ([1987] 2001). L'apprentissage de l'abstraction. Paris : Retz.

BECK U. ([1986] 2001). La société du risque. Sur la voie d'une autre modernité. Paris : Flammarion.

BODEN M. A. (2005). The creative mind. Myths and mechanisms. New York: Routledge, $2^{\mathrm{e}}$ éd.

BRUNER J. ([1996] 2008). L'éducation, entrée dans la culture. Paris : Retz.

CALLON M., LASCOUMES P. \& BARTHE Y. (2001). Agir dans un monde incertain. Essai sur la démocratie technique. Paris : Éd. du Seuil. 
CITTON Y. (2010). L'avenir des Humanités. Économie de la connaissance ou cultures de l'interprétation? Paris : La Découverte.

CITTON Y. \& LATOUR B. (2013). «Anthropologie du Futur/Crise du Futur». La suite dans les idées, 7 septembre 2013. En ligne : <https://www.franceculture.fr/emissions/la-suitedans-les-idees/anthropologie-du-futur-crise-du-futur $>$ (consulté le 28 février 2018).

DI MÉO G. \& BULÉON P. (2005). L'espace social. Lecture géographique des sociétés. Paris : Armand Colin.

ELIAS N. (1993). Qu'est-ce que la sociologie? Paris : Pocket.

FABRE M. (2009). Philosophie et pédagogie du problème. Paris : Vrin.

FABRE M. (2011). Éduquer pour un monde problématique. La carte et la boussole. Paris : PUF.

FRISCHKNECHT-TOBLER U., NAGEL U. \& SEYBOLD H. E. (2008). Systemdenken. Wie Kinder und Jugendliche Komplexe Systeme verstehen lernen. Zurich : Verlag Pestalozzianum an der Pädagogischen Hochschule Zurich.

GAUDIN T. (dir.) (1990). 2100, récit du prochain siècle. Paris : Payot.

HERTIG P. (2009). Didactique de la géographie et formation initiale des enseignants spécialistes. Conception et première évaluation du nouveau dispositif de formation initiale des enseignants de géographie du Secondaire supérieur à la HEP Vaud. Thèse de doctorat, géosciences et sciences de l'environnement, Université de Lausanne.

HERTIG P. (2011). «Le développement durable : un projet multidimensionnel, un concept discuté». In A. Pache, P.-P. Bugnard \& P. Haeberli (dir.), Éducation en vue du développement durable. École et formation des enseignants : enjeux, stratégies et pistes. Neuchâtel : CDHEP, p.19-38.

HUMBEL L., JOLLIET F. \& VARCHER P. (2013). «La déconstruction et l'élément déclencheur, deux démarches-clés pour permettre le développement d'un apprentissage fondamental en EDD : la capacité de problématiser. Une application en classes de collège au sujet de QSV liées au fait religieux». Penser l'éducation, hors série, p.329-345.

JANZI H. \& SGARD A. (2013). "Le "savoir des questions" : comment problématiser avec les élèves? Un exemple d'élément déclencheur : des éoliennes dans le paysage genevois ». Penser l'éducation, hors série, p. 205-221.

JENNI P., VARCHER P. \& HERTIG P. (2013). «Des élèves débattent : sont-ils en mesure de penser la complexité?». Penser l'éducation, hors série, p.187-203.

LANGE J.-M. (dir.). (2013). Penser l'éducation. Philosophie de l'éducation et Histoire des idées pédagogiques. Actes du Colloque international «L'éducation au développement durable : appuis et obstacles à sa généralisation hors et dans l'École», université de Rouen.

LAPLACE C. (2006). «Problématisation, conseils d'élèves et formation des enseignants ». In M. Fabre \& E. Vellas (dir.), Situations de formation et problématisation. Bruxelles : De Boeck \& Larcier, p. 159-174.
LEBATTEUX N. \& LEGARDEZ A. (2011). «Rapport aux savoirs sur le développement durable en contexte scolaire : obstacles à la mise en œuvre d'un Agenda 21 en France». In A. Pache, P.-P. Bugnard \& P. Haeberli (dir.), Éducation en vue du développement durable. École et formation des enseignants : enjeux, stratégies et pistes. Neuchâtel : CDHEP, p.179-199.

LUBART T. (2012). «Les ressorts psychologiques de la créativité ». Revue économique et sociale, n², p. 13-21.

LUBART T., MOUCHIROUD C., TORDJAM S. \& ZENASNI F. (2003). Psychologie de la créativité. Paris : Armand Colin.

LUSSAULT M. (2007). L'Homme spatial. Paris : Éd. du Seuil.

MARKOVA I. (2003). "Les focus groups». In S. Moscovici \& F. Buschini (dir.), Les méthodes des sciences humaines. Paris : PUF, p. 221-242

MORIN E. (1999). La tête bien faite. Repenser la réforme, réformer la pensée. Paris : Éd. du Seuil.

MORIN E. (2000). Les sept savoirs nécessaires à l'éducation du futur. Paris : Éd. du Seuil.

MUSSET M. (2010). "L'éducation au développement durable». Dossier d'actualité de la VST, $\mathrm{n}^{\circ} 56$. En ligne : $<$ http://ife.ens-lyon.fr/vst/LettreVST/SommaireLettre. htm $>$ (consulté le 28 février 2018).

ORANGE-RAVACHOL D. \& DOUSSOT S. (2013). «Engager l'école dans l'EDD risque-t-il de la dédisciplinariser? ».Penser l'éducation, hors série, p. 81-96.

OSSIMITZ G. (2000). Entwicklung systemischen Denkens. Theoretische Konzepte und empirische Untersuchungen. Vienne (Autriche); Munich (Allemagne) : Profil Verlag.

PACHE A. (2014). L'alimentation à l'école. Enseigner une géographie renouvelée. Rennes : Presses universitaires de Rennes.

PACHE A., BUGNARD P.-P. \& HAEBERLI P. (dir.) (2011). Éducation en vue du développement durable. École et formation des enseignants: enjeux, stratégies et pistes. Neuchâtel : CDHEP.

PERRIN N. (2014). «Créativité : il est autorisé d'interdire». L'Éducateur, n², p. 10.

PUOZZO CAPRON I. \& MARTIN D. (2014). «De la pensée créatrice à la pensée créative». L'Éducateur, n², p.13-14.

REMPFLER A. (2009). «Systemkompetenz: Forschungstand und Forschungsfragen ». Geographie und ihre Didaktik, n०37(2), p.58-79.

REMPFLER A. (2010). «Systemdenken - Schlüsselkompetenz für zukunftsorientiertes Raumverhalten ». Geographie und Schule, no32(184), p.11-18.

REMPFLER A. \& UPHUES R. (2012). "System Competence in Geography Education. Development of competence models, diagnosing pupil's achievement ». European Journal of Geography, n³(1), p. 6-22.

SCHUMACHER A., COEN P.-F. \& STEINER M. (2010). «Les futurs enseignants et la créativité : quelles conceptions?». Formation et pratiques d'enseignement en questions, $\mathrm{n}^{\circ} 11$, p. 115-131. 
STERLING S. (2011). «Transformating Learning and Sustainability: sketching the conceptual ground ». Learning and Teaching in Higher Education, ${ }^{\circ}{ }^{5}$, p. 17-33.

TUTIAUX-GUILLON N. (2008). "Apprentissages socioculturels et apprentissages disciplinaires en histoiregéographie». Les Cahiers Théodile, n 9, p.61-78.

VARCHER P. (2011). «L'éducation en vue du développement durable : une filiation à assumer, des défis à affronter $»$. In F. Audigier, N. Fink, N. Freudiger \& P. Haeberli (dir.),
L'éducation en vue du développement durable : sciences sociales et élèves en débats. Genève : Université de Genève, p.25-46.

VERGNOLLE MAINAR C. (2011). La géographie dans l'enseignement. Une discipline en dialogue. Rennes : Presses universitaires de Rennes.

WELZER H. (2009). Les guerres du climat. Pourquoi on tue au $X X I^{e}$ siècle. Paris : Gallimard. 